\title{
Cardiac reflections of attention and preparatory set in a chimpanzee (Pan troglodytes)
}

\author{
GARY G. BERNTSON and SARAH T. BOYSEN \\ Ohio State University, Columbus, Ohio \\ and Yerkes Regional Primate Research Center, Emory University, Atlanta, Georgia
}

\begin{abstract}
Tonic and phasic measures of heart rate were obtained during vigilance performance in a chimpanzee. Results revealed that patterns of cardiac activity are correlated with critical dimensions of performance, and are highly comparable to those of human subjects. Baseline heart rate decreased within individual sessions and increased over sessions, in close correspondence to progressive trends in reaction time. Heart period variability also covaried with reaction time, faster reaction time being associated with both higher heart rate and lower heart period variability. Phasic patterns of cardiac activity, however, appeared to relate more specifically to performance variables than did baseline measures. Target detection was followed by notable cardiac acceleration, whereas misses, correct rejections, and false alarms were associated with heart rate decelerations. The cardiac acceleration to target detection appeared not to result simply from a feedback consequence of the motor response, but rather to reflect aspects of the detection process. Finally, target stimuli were detected more frequently when they occurred after a long heart period. This did not reflect an effect of baseline heart rate, since the probability of target detection did not covary with changes in baseline heart rate. Rather, the results are more consistent with the view that temporal fluctuations in an attentional or preparatory state are reflected in transient variations in heart period.
\end{abstract}

Processes involved in sustained attention have been extensively studied in vigilance tasks, which require the detection of target stimuli embedded within a stream of nontarget events. Although considerable data are now available on task variables and performance determinants of vigilance, many diverse theoretical perspectives still exist, and no single comprehensive theory of vigilance has yet achieved widespread acceptance (Parasuraman \& Davies, 1984; Warm, 1984). Psychophysiological measures obtained during task performance may offer insights into the nature and dynamics of the cognitive events underlying attentional performance, and thereby contribute to the evaluation of alternative theoretical accounts. Earlier studies of heart rate and electrodermal responses offered some general support for arousal theories of vigilance and vigilance decrements, but at the same time revealed serious limitations of arousal explanations of sustained attention (Parasuraman, 1984a, 1984b). These early studies, however, generally focused on tonic levels of autonomic activity over relatively long blocks of time (e.g., Mackie, 1977), and it is unlikely that such gross measures of autonomic tone could provide the specificity of information required for the development of refined theoretical

This research was supported in part by a grant from Ohio State University and in part by NIH Grant RR-00165 from the Division of Research Resources to the Yerkes Regional Primate Research Center. The Yerkes Center is fully accredited by the American Association for Laboratory Animal Care. We acknowledge the continued cooperation of Jack Hanna, Director of the Columbus Zoo. Requests for reprints should be sent to G. G. Berntson, 48 Townshend Hall, Ohio State University, 1885 Neil Ave., Columbus, OH 43210-1222. models. A finer temporal analysis of autonomic activity, however, may provide more critical information on the cognitive events underlying sustained attention. Blakeslee (1979), for example, observed an increase in electrodermal responses immediately prior to target detection, but no such increase when targets were missed, suggesting that temporally fluctuating anticipatory or preparatory states may be associated with efficient vigilance performance.

As part of an ongoing evaluation of cognitive processes in the chimpanzee, we recently completed an initial study of vigilance performance in this species (Berntson, Boysen, \& Long, 1987), and have characterized its basic patterns of cardiac response to significant and nonsignal stimuli (Berntson \& Boysen, 1984, 1987). To further examine attentional processes, we measured tonic and phasic cardiac adjustments during vigilance performance in a chimpanzee that had participated in the previous study. Specifically, we examined the degree to which cardiac measures reflect the cognitive and performance aspects of vigilance in the chimpanzee, and the extent to which these cardiac measures correspond to those reported for humans.

\section{METHOD}

\section{Subject}

The subject, Sheba, was a 4.5-year-old female captive-born chimpanzee. Sheba had been home reared for the first 2 years of life, and had participated in the Primate Cognition Project at Ohio State University for approximately 24 months prior to the present study. During her involvement in the project, Sheba had been interactively 
trained on a variety of cognitive skills, and had participated in an earlier study of attentional performance, which afforded her considerable practice on vigilance tasks.

\section{Apparatus}

The testing apparatus consisted of a Lexan-covered rack-mount cabinet with a video monitor for stimulus presentation, and a Plexiglas response panel $(7.6 \times 10.2 \mathrm{~cm})$ centered beneath the monitor screen. A Lexan-covered plastic cup (10 cm in diameter) was secured below and to the side of the response panel, and served as a receptacle for reinforcements presented via a delivery tube. A microcomputer system controlled stimulus presentations and timing, and also recorded responses. The stimuli for the vigilance task consisted of a single nontarget stimulus (a white square, $7.5 \times 7.5 \mathrm{~cm}$ on a black background) and a single target stimulus (a white cross with equivalent horizontal and vertical dimensions). Both stimuli subtended approximately $10^{\circ}$ of visual angle, with a figure luminance of $260 \mathrm{~lx}$, and a background luminance of $<5 \mathrm{~lx}$.

\section{Procedure}

Prior to the present study, Sheba had participated in a larger study on vigilance performance in chimpanzees, and was well practiced in the vigilance task (Berntson, Boysen, \& Long, 1987). The present procedure differed in that reinforcers, although they were delivered during task performance, remained within the covered receptacle and were unavailable until the end of the session. This change was implemented to eliminate potentially confounding effects of consummatory responses on the cardiac measures. Individual sessions consisted of the presentation of 100 stimuli with a $50 \%$ target probability (interstimulus interval $=5.0 \mathrm{sec}$; total session duration = $8.3 \mathrm{~min}$ ). Each correct response (panel press to the target stimulus) was followed by the delivery of reinforcement (candy) into the covered food well. The task stimuli were terminated when a response occurred or at a maximum of $3.5 \mathrm{sec}$ after onset, at which point reinforcement was no longer available. Following training on the new reinforcement regimen, five additional preliminary sessions were given to further stabilize performance and to minimize potential frustration effects associated with the lack of immediate reward. All training and testing were completed prior to the main daily meal, but no explicit food deprivation was employed.

Following training, Sheba was given nine formal test sessions during which she was isolated in a $1 \mathrm{~m} \times 1 \mathrm{~m}$ area bounded by the testing apparatus on one side and by solid walls and a wooden barrier on the remaining sides. The testing space served to minimize distraction, but was not intended to physically restrain the animal, since her active participation was essential for the acquisition of meaningful data. Consequently, the top of the testing area was not enclosed, and the animal could exit the testing situation. There was no interaction between the animal and the experimenter during testing, unless Sheba attempted to leave the testing space. In this case she was instructed, verbally and with gestures, to remain within the testing booth. Had she failed to comply, testing would have been discontinued for that session, but this never happened.

\section{EKG Measures}

Heart rate was recorded noninvasively during task performance, to evaluate tonic heart rate changes and phasic responses to the task stimuli. Silver/silver chloride EKG electrodes were attached to thoracic monitor sites and secured by an elastic band. The EKG signal was amplified by an Amerec ERM 101 cardiotachometer and was recorded, along with a stimulus marker, on a Burdick FM cardiocassette recorder. The $\mathrm{R}$-wave-detector output from the cardiotachometer was coupled to a microprocessor data acquisition system for the on-line determination of individual heart periods. The FM tape was subsequently played back on a polygraph (Grass Model 7, at $30 \mathrm{~mm} / \mathrm{sec}$ ) for a permanent record and for artifact editing. Potential artifacts in the heart period data were identified off-line by the microcomputer system as periods that deviated from either of the immediately adjacent beats by more than $30 \%$. Heart periods exceeding this criteria were checked and were corrected, if necessary, by direct measurement from the polygraph record.

\section{Data Analysis}

Reaction time, probability of hits (correct detection of the target), and probability of false alarms (responses to a nontarget stimulus) served as the primary performance measures. Chi-square tests for goodness of fit, with the Yates correction, were used to evaluate differences in the probabilities of hits, false alarms, and responses across experimental conditions. Friedman's test and the Wilcoxon $T$ test were used to evaluate differences in reaction time over sessions or session blocks (Hays, 1981). Performance across sessions was also evaluated by signal detection analyses to provide independent estimates of perceptual sensitivity and response criterion. Nonparametric indices of signal detection parameters were derived as suggested by Craig (1979). These included a measure of perceptual sensitivity $\left(A^{\prime}\right)$ that corresponds to the parametric index $d^{\prime}$, and a measure of response criterion $\left(B^{\prime}\right)$ that corresponds to the parametric index $\beta . A^{\prime}$ values can range from .5 (no discriminative sensitivity) to 1 (high perceptual sensitivity), and $B^{\prime}$ values range from -1 (low response criterion) to +1 (high criterion). These indices were derived primarily for descriptive purposes, however, and all statistical analyses were based on performance dimensions as described above. The small number of false alarms precluded meaningful application of signal detection measures to within-session variations in performance.

To minimize contamination from phasic cardiac responses to the task stimuli, measures of baseline heart rate were based on the prestimulus heart periods throughout the course of the sessions. The analysis of phasic cardiac responses to the task stimuli was based on the single prestimulus heart period and the subsequent poststimulus beats (over the 5 -sec interstimulus interval). In addition, a measure of heart period change was calculated as the mean poststimulus heart period minus the mean prestimulus period. Finally, a measure of baseline heart period variability was calculated as the standard deviation of all heart periods over the relevant interval. For comparisons of phasic variability changes among different stimuli or trial outcomes, we also employed the successive-difference mean square statistic for the poststimulus heart periods (Heslegrave, Ogilvie, \& Furedy, 1979). This statistic was selected because of its minimal sensitivity to nonlinear trends in heart periods, such as would be expected to occur in response to task stimuli.

Heart period data were analyzed separately for the following classes of trials: hits, misses, correct rejections, and false alarms. Because of the single-subject design, all analyses were by nonparametric methods. Friedman's analysis of variance was used to identify overall differences in heart periods or heart period change over multiple trial classes or conditions, and Wilcoxon $T$ tests were used for paired comparisons. All analyses were based on heart periods, although these data were sometimes converted to rate for clarity of exposition. The small number of false alarms observed ( 7 out of 450 opportunities) precluded statistical analyses of cardiac responses for this class of trials, but these data are presented for illustration.

\section{RESULTS}

\section{Vigilance Performance}

Overall vigilance performance is illustrated in Figure 1, which shows within-session performance trends over blocks of trials and across-sessions trends over session blocks. In general, Sheba displayed highly stable attentional performance over sessions, although her reaction 

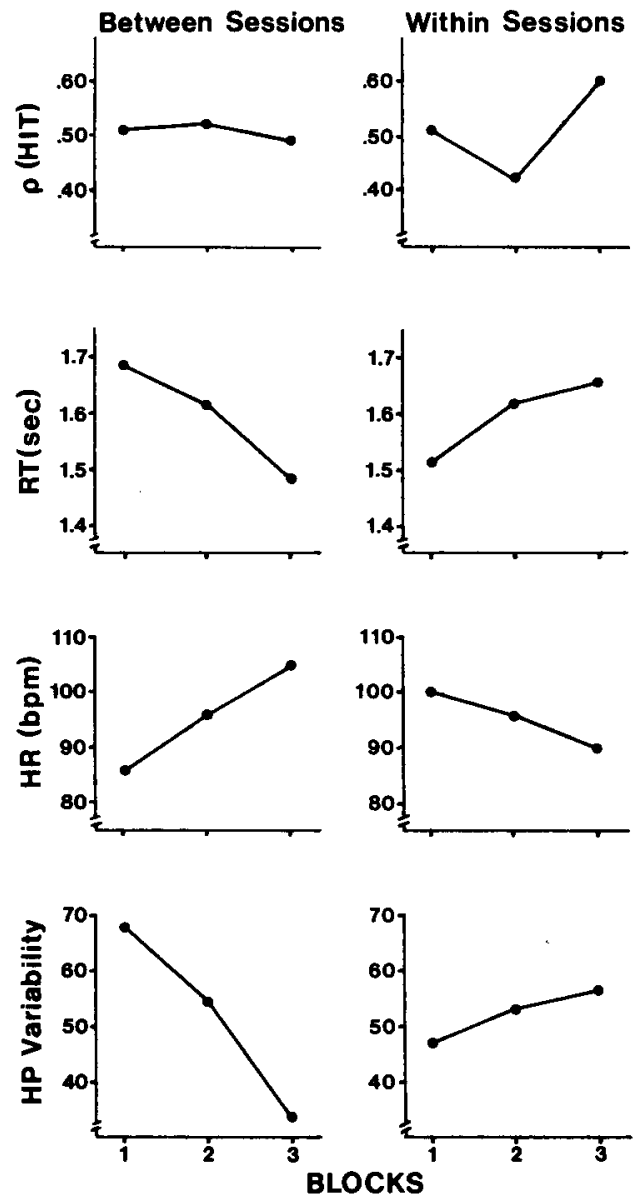

Figure 1. Performance characteristics and baseline cardiac measures over three within-session and between-sessions blocks. (Withinsession blocks comprise 33, 34, and 33 trials, respectively. Fach session block consists of the mean of three consecutive test sessions.)

time progressively decreased. Sheba had previously demonstrated a moderate perceptual sensitivity and a high response criterion in vigilance tasks (relative to those of other animals), resulting in a low rate of false alarms as well as a relatively low hit rate. Her response criterion was even higher in the present study, and she achieved a very low hit rate (Table 1 ). This was probably attributable to the absence of within-session reinforcement in the present study.

Although Sheba's overall error rate was relatively stable across sessions, systematic within-session changes were apparent. To evaluate these trends in performance, data were analyzed over three successive blocks of trials (containing 33, 34, and 33 trials). As illustrated in Figure 1, a typical within-session vigilance decrement was manifested by a progressive increase in reaction time over trial blocks [Friedman $\chi_{r}^{2}(2)=6.22, p<.05$ ]. Moreover, a significant within-session variation in hit rate was also apparent $\left[\chi^{2}(2)=9.51, p<.01\right]$, reflecting a vigilance decrement from the first to the second block $\left[\chi^{2}(1)=3.92, p<.05\right]$. Detection rate improved again, however, in the last block $\left[\chi^{2}(1)=8.80, p<.01\right]$. This late-appearing reversal of the vigilance decrement may have reflected an incentive effect associated with the upcoming availability of the reinforcers earned during the session.

\section{Baseline Heart Rate}

Baseline heart rate during the course of the sessions showed a general correspondence with task performance. As illustrated in Figure 1, the decreasing reaction time over sessions was paralleled by an increase in heart rate, and increasing reaction time within the individual sessions was associated with a corresponding decrease in baseline heart rate. Over all sessions and trial blocks, baseline heart rate and reaction time were significantly correlated $(r=-.47, p<.02)$.

\section{Momentary Heart Rate}

The hit rate, in contrast to reaction time, did not appear to covary with baseline heart rate over trial blocks $(r=.02)$. There did emerge, however, a specific statistical association between the immediate prestimulus heart period and the probability of target detection. Table 2 summarizes the characteristics of the prestimulus beats for different stimuli and trial outcomes. The means and distributions of prestimulus beats that occurred prior to nontarget stimuli did not differ from those of beats that occurred prior to target stimuli. Target detection, however, was superior when the stimulus fell after a long heart period, and was inferior when the stimulus occurred after a fast beat. Thus, prestimulus beats associated with detected targets were significantly longer than those associated with missed targets [Wilcoxon $T(9)=6$, $p<.05]$. This relationship is further reflected by a significantly increasing hit rate for target stimuli that fell after progressively longer prestimulus heart periods [probability of a hit after prestimulus heart periods ranging from 301 to $550 \mathrm{msec}=.45 ; 551$ to $750 \mathrm{msec}=.52 ; 751$ to $950 \mathrm{msec}=.64 ; \chi^{2}(2)=7.38, p<.03$ ]. Prestimulus heart periods were also longer prior to false alarms, although their small number precluded statistical evaluation.

\section{Phasic Cardiac Response}

The overall cardiac responses to the stimuli of the vigilance task are illustrated in Figure 2 . Correct detections, missed targets, and false alarms were associated with poststimulus increases in heart period (cardiac decelerations). In contrast, the detection of target stimuli

Table 1

Vigilance Performance Across Session Blocks

\begin{tabular}{ccccccc}
\hline Session & RT (msec) & $p(\mathrm{R})$ & $p($ Hit $)$ & $p($ FA) & $A^{\prime}$ & $B^{\prime}$ \\
\hline $1-3$ & 1685 & .27 & .51 & .02 & .87 & .92 \\
$4-6$ & 1549 & .26 & .52 & .01 & .88 & .97 \\
$7-9$ & 1484 & .25 & .49 & .02 & .86 & .92 \\
Mean & 1574 & .26 & .51 & .02 & .87 & .94 \\
\hline
\end{tabular}

Note $-\mathbf{R}=$ response; $F A=$ false alarm. 
Table 2

Characteristics of the Prestimulus Heart Periods for Different Stimuli and Outcomes

\begin{tabular}{lccccr}
\hline & $\begin{array}{c}\text { Number of } \\
\text { Stimuli }\end{array}$ & $\begin{array}{c}\text { Mean } \\
(\mathrm{msec})\end{array}$ & $\begin{array}{c}\text { Median } \\
(\mathrm{msec})\end{array}$ & \multicolumn{1}{c}{ SD } & SE \\
\hline Targets & 450 & 628.9 & 589 & 130.8 & 6.2 \\
Misses & 222 & 617.4 & 581 & 129.2 & 8.7 \\
Hits & 228 & 640.0 & 597 & 131.7 & 8.7 \\
Nontargets & 450 & 627.2 & 590 & 121.8 & 5.8 \\
$\quad$ Correct rejections & 443 & 626.9 & 589 & 121.9 & 5.8 \\
False alarms & 7 & 642.9 & 628 & 125.5 & 51.2 \\
\hline
\end{tabular}

was associated with a notable cardiac acceleration $(11 \mathrm{bpm})$, reflected by a significant poststimulus decrease in heart periods [Wilcoxon $T(9)=2, p<.02]$. As indicated above, no overall differences were observed between the prestimulus heart periods associated with target stimuli and those associated with nontarget stimuli, although prestimulus heart periods for detected targets were longer than those for missed targets. The differential pattern of cardiac response to detected targets, however, was not a statistical artifact of the longer prestimulus beats. Significant differences among trial classes (hits, misses, correct rejections) were apparent not only in the magnitude of heart period change from baseline [Friedman $\left.\chi_{,}^{2}(2)=8.67, p<.02\right]$, but in the absolute poststimulus heart period level [Friedman $\chi_{r}^{2}(2)=8.22$, $p<.02]$. Poststimulus heart periods were significantly shorter after target detections than after either missed targets [Wilcoxon $T(9)=3, p<.02$ ] or correct rejections of nontarget stimuli [Wilcoxon $T(9)=1, p<.01$ ]. In contrast, cardiac responses after missed targets and after correct rejections did not differ from each other. Thus, although the prestimulus beats were longer before target

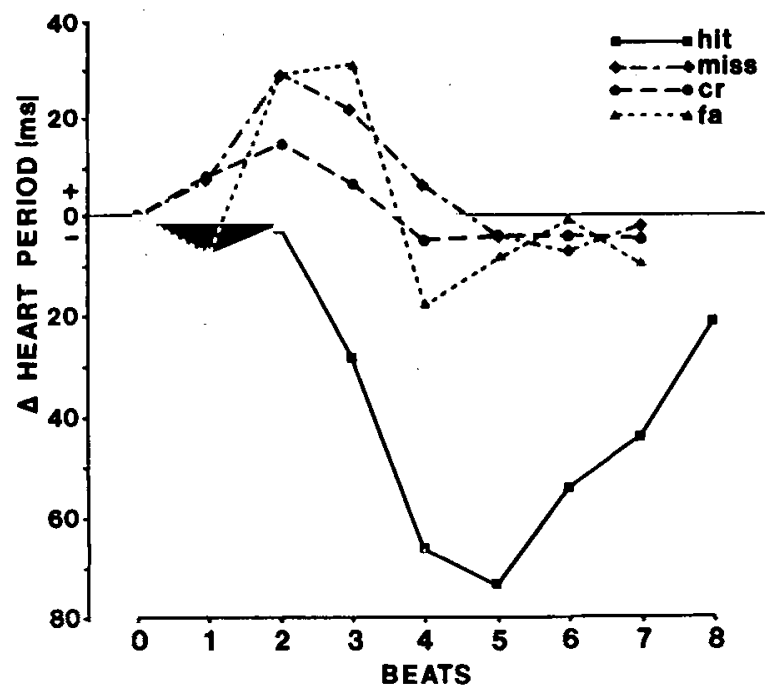

Figure 2. Heart period responses to task stimuli for detected targets, missed targets, correct rejections, and false alarms, over the 5 -sec interstimulus interval. Data points represent beat-by-beat heart periods relative to prestimulus baseline. Stimuli were presented during beat 1 . (The additional data point for target detections is due to the short heart periods under this condition.) detections, the poststimulus periods were significantly shorter than for other trial outcomes.

The cardiac acceleration associated with target detection did not appear to simply reflect the feedback consequences of the motor response, since cardiac deceleration was observed with similar responses to nontarget stimuli (false alarms). Although the small number of false alarms (7) precluded statistical comparisons, the cardiac responses on these trials were highly comparable to those for misses and correct rejections (Figure 2).

\section{Heart Period Variability}

Heart period variability, as indexed by the standard deviation of the beats, was correlated with baseline heart rate $(r=-.73, p<.01)$; higher heart rate was associated with lower heart period variability. This variability measure therefore followed the general withinsession and between-sessions trends in baseline heart rate, showing a general increase within sessions, and an overall decrease across sessions. Consequently, baseline heart period variability was also significantly correlated with reaction time over session blocks $(r=.58, p<.02)$. Further analysis of beat-by-beat changes in variability, indexed by the successive-difference mean square statistic (Heslegrave et al., 1979), revealed that heart period variability significantly decreased after all stimulus presentations [Wilcoxon $T(9)=3, p<.02$ ]. The magnitude of this stimulus-evoked decrease in variability did not differ across stimulus types (target or nontarget) or trial outcomes (hits, misses, or correct rejections).

\section{DISCUSSION}

The present results reveal that cardiac responses are associated with specific dimensions of vigilance performance in the chimpanzee. Studies on human subjects have indicated that attentional performance is related to psychophysiological measures that index arousal, including baseline heart rate, heart rate variability, electrodermal activity, and EEG activity (Blakeslee, 1979; Griew, Davies, \& Treacher, 1963; O’Hanlon \& Beatty, 1977; Parasuraman, 1984a, 1984b; Porges, 1984; Thackray, Bailey, \& Touchstone, 1977). The present results are in accord with these findings. Baseline heart rate decreased within individual sessions and increased over sessions, inversely corresponding with progressive trends in reaction time. Similarly, heart period variability correlated with reaction time; faster reaction times were associated with lower heart period variability. This is consistent with previous vigilance studies (Griew et al., 1963; O'Hanlon \& Beatty, 1977 ) and with reports that superior performance in other cognitive tasks is associated with a reduction in heart period variability (Mulder \& Mulder, 1981; Porges, 1984). It is not likely that arousal is a unitary process, however, and the relationship between arousal measures and attentional performance is at best complex. Although the level of behavioral activation can certainly affect vigilance, arousal theories have not been particularly 
robust in accounting for the quantitative features of attentional performance (Mackworth, 1970; Parasuraman, 1984a, 1984b).

As previously observed for pupillary responses (Beatty, 1982a), phasic patterns of cardiac activity in the present study appeared to be more closely related to performance variables than were baseline measures. Target detection was followed by notable cardiac acceleration, whereas misses and correct rejections were associated with heart rate deceleration. The cardiac acceleration following target detection did not appear to reflect simply the motor demands of the response, since a contrasting cardiac deceleration was observed following false alarms, in which case the same response was emitted to nontarget stimuli. Rather, the cardiac acceleration probably reflected some aspect of the detection process, or an interaction between target detection and response processes. These results are consistent with previous reports that target detection in humans is often associated with postevent cardiac acceleration (Jennings, 1984; Porges, 1972), pupillary dilation (Beatty, 1982b), and enhanced electrodermal activity (Blakeslee, 1979). Because cardiac orienting responses to nonsignal stimuli have generally been found to be deceleratory in both humans and apes (Berntson \& Boysen, 1984; Graham, 1979; Turpin, 1983), the cardiac acceleration observed after target detection does not appear to parallel the orienting response to nonsignal stimuli. Although the origin of this cardiac acceleration remains to be clarified, it has become increasingly apparent that several factors associated with signal detection, including response-decision processes and incentive effects (Fowles, Fisher, \& Tranel, 1982; Obrist et al., 1974; Spinks \& Siddle, 1983), may contribute to acceleratory responses. Although changes in somatic activity may also have contributed to the cardiac acceleration, two considerations argue against this possibility. First, notable somatic responses were not apparent after the delivery of reinforcers following target detection. Second, even clear somatic responses (panel pressing to negative stimuli) were not sufficient to induce cardiac acceleration on false alarm trials.

Perhaps more significant than the postevent responses are the apparent prestimulus cardiac features that preceded target detection. Considerable interest has been devoted to the impact of preparatory states on cognitive, perceptual, and motor processes in human performance (Kornblum \& Requin, 1984). Moreover, considerable empirical data now support a relationship between autonomic activity and anticipatory states and processes in human cognitive performance (Coles, 1984; Spinks, Blowers, \& Shek, 1985; Spinks \& Siddle, 1983). A common experimental method for the study of preparatory processes is the two-stimulus reaction time paradigm, in which a warning or conditioning stimulus is introduced prior to an imperative stimulus. Studies employing this paradigm have revealed a variety of systematic psychophysiological adjustments that occur in the interstimulus interval and are predictive of subsequent performance. These include cardiac responses (Coles, 1984; Jennings, 1984; Lacey \& Lacey, 1974; Spinks \& Siddle, 1983), as well as cerebral event-related potentials such as the contingent negative variation, readiness potential, and slow wave (Donchin, Coles, \& Gratton, 1984; Evarts, 1984). Although the two-stimulus paradigm provides an explicit warning signal that allows for time-locked psychophysiological measures, it is unlikely that modulations of preparatory states occur only in response to experimental stimuli. Indeed, endogenously fluctuating preparatory states may be significant determinants of vigilance performance. Jerison (1970) suggested that endogenously generated "observing responses"' can contribute to target detection, and Maltzman (1970) hypothesized similar internally generated "orienting responses."

This view is consistent with reports of increased electrodermal activity prior to detected targets, over that prior to missed targets, in vigilance tasks (Blakeslee, 1979; Surwillo \& Quilter, 1965). It is also in keeping with the finding of the present study that target stimuli were detected more frequently when they occurred after a long heart period. This finding was not related to an influence of baseline heart rate, inasmuch as the probability of target detection did not covary with absolute changes in tonic heart rate, either within or between sessions. Rather, the present results are more consistent with the view that temporal variations in attentional or preparatory states are associated with central processes that are reflected in momentary fluctuations of baseline heart rate. It is not clear whether the antecedent state was associated with enhanced sensory processing (Lacey \& Lacey, 1974) or reflected response preparation (Jennings, 1984; Obrist et al., 1974). The longer heart periods observed prior to false alarms, however, raise the possibility that the antecedent state may reflect a component of response preparation.

In summary, the present data suggest that patterns of autonomic response during vigilance performance in the chimpanzee are highly comparable to those of human subjects. In addition to a general association between baseline heart rate and reaction time, two specific relationships emerged in the present study between cardiac measures and target detection. The first is associated with a prestimulus preparatory (or anticipatory) state that favors target detection and is characterized by a slow momentary heart rate. The second is a postevent response, characterized by cardiac acceleration, that follows target identification. Until recently, autonomic studies of vigilance performance have generally focused on baseline measures of general arousal. The present results suggest that a finer temporal analysis of psychophysiological parameters may prove more fruitful in the evaluation of cognitive processes underlying sustained attention. Although the generality of the present results is limited by the single-subject design, the apparent existence of common autonomic markers of vigilance performance in 
chimpanzees and humans offers a promising approach to the comparative study of attention.

\section{REFERENCES}

Beatty, J. (1982a). Phasic not tonic pupillary responses vary with auditory vigilance performance. Psychophysiology, 19, 167-172.

BeAtTY, J. (1982b). Task-evoked pupillary responses, processing load, and the structure of processing resources. Psychological Bulletin, 91, 276-292.

Berntson, G. G., \& Boysen, S. T. (1984). Cardiac startle and orienting responses in the Great Apes. Behavioral Neurosciences, 98, 914-918.

Berntson, G. G., \& Boysen, S. T. (in press). Cardiac correlates of cognition in infants, children and chimpanzees. In L. P. Lipsitt \& C. Rovee-Collier (Eds.), Advances in infancy research. Norwood, NJ: Ablex.

Berntson, G. G., Boysen, S. T., \& Long, G. (1987). Vigilance: Attentional performance in chimpanzees (Pan troglodytes). Manuscript submitted for publication.

BlaKeSLEe, P. (1979). Attention and vigilance: Performance and skin conductance response changes. Psychophysiology, 16, 413-419.

ColEs, M. G. H. (1984). Heart rate and attention: The intake-rejection hypothesis and beyond. In M. G. H. Coles, J. R. Jennings, \& J. A. Stern (Eds.), Psychophysiological perspectives: Festschrift for Beatrice and John Lacey (pp. 276-311). New York: Van Nostrand Reinhold.

CraIg, A. (1979). Nonparametric measures of sensory efficiency for sustained monitoring tasks. Human Factors, 21, 69-78.

Donchin, E., Coles, M. G. H., \& Gratton, G. (1984). Cognitive psychophysiology and preparatory processes: A case study. In S. Kornblum \& J. Requin Preparatory states and processes (pp. 155-178). Hillsdale, NJ: Erlbaum.

EvarTs, E. V. (1984). Neurophysiological approaches to brain mechanisms for preparatory set. In S. Kornblum \& J. Requin (Eds.), Preparatory states and processes (pp. 155-178). Hillsdale, NJ: Erlbaum.

Fowles, D. C., Fisher, A. E., \& Tranel, D. T. (1982). The heart beats to reward: The effect of monetary incentive on heart rate. Psychophysiology, 19, 506-513.

GraHAM, F. (1979). Distinguishing among orienting, defensive, and startle reflexes. In H. D. Kimmel, E. H. van Olst, J. F. Orlebeke (Eds.), The orienting reflex in humans (pp. 137-167). Hillsdale, NJ: Erlbaum.

Griew, S., Davies, D. R., \& Treacher, A. (1963). Heart rate and auditory vigilance performance. Nature, 200, 1026.

HAYs, W. L. (1981). Statistics. New York: Holt, Rinehart \& Winston.

Heslegrave, R. J., Ogilvie, J. C., \&uRedy, J. J. (1979). Measuring baseline-treatment differences in heart rate variability: Variance versus successive difference mean square and beats per minute versus interbeat intervals. Psychophysiology, 16, 151-157.

JENNINGS, J. R. (1984). Cardiac-cycle time, sensorimotor integration, and motor impulsivity: A fifties revival. In M. G. H. Coles, J. R. Jennings, \& J. A. Stern (Eds.), Psychophysiological perspectives: Festschrift for Beatrice and John Lacey (pp. 260-275). New York: Van Nostrand Reinhold.

JERISON, H. J. (1970). Vigilance, discrimination, and attention. In D. I. Mostofsky (Ed.), Attention: Contemporary theory and analysis (pp. 127-147). New York: Appleton-Century-Crofts.

Kornblum, S., \& ReQuin, J. (EDS.). (1984). Preparatory states and processes. Hillsdale, NJ: Erlbaum.
LACEY, B. C., \& LACEY, J. I. (1974). Studies of heart rate and other bodily processes in sensorimotor behavior. In P. A. Obrist, A. H. Black, J. Brener, \& L. V. DiCara (Eds.), Cardiovascular psychophysiology: Current issues in response mechanisms, biofeedback, and methodology (pp. 538-564). Chicago: Aldine.

MACKIE, R. (ED.) (1977). Vigilance: Theory, operational performance, and physiological correlates. New York: Plenum Press.

MACKWORTH, J. F. (1970). Vigilance and attention. Baltimore: Penguin. Maltzman, I. (1970). The orienting reflex and thinking as determiners of conditioning and generalization to words. In H. H. Kendler \& J. T. Spence (Eds.), Essays in neobehaviorism: A memorial volume to Kenneth $W$. Spence (pp. 89-111). New York: Appleton-Century-Crofts.

MULDER, G., MULDER, L. J. M. (1981). Information processing and cardiovascular control. Psychophysiology, 18, 392-401.

Obrist, P. A., Howard, J. L., Lawler, J. E., Galosy, R. A., MEYers, K. A., \& GaEbelein, C. J. (1974). The cardiac-somatic interaction. In P. A. Obrist, A. H. Black, J. Brener, \& L. V. DiCara (Eds.), Cardiovascular psychophysiology: Current issues in response mechanisms, biofeedback, and methodology (pp. 136-162). Chicago: Aldine.

O'Hanlon, J. F., \& BeATty, J. (1977). Concurrence of electroencephalographic and performance changes during a simulated radar watch and some implications for the arousal theory of vigilance. In R. Mackie (Ed.), Vigilance: Theory, operational performance, and physiological correlates (pp. 189-202). New York: Plenum Press.

Parasuraman, R. (1984a). The psychobiology of sustained attention. In J. S. Warm (Ed.), Sustained attention in human performance (pp. 61-101). New York: Wiley.

Parasuraman, R. (1984b). Sustained attention in detection and discrimination. In R. Parasuraman \& D. R. Davies (Eds.), Varieties of attention (pp. 243-272).

Parasuraman, R., \& Davies, D. R. (Eds.). (1984). Varieties of attention. New York: Academic Press.

Porges, S. W. (1972). Heart rate variability and deceleration as indexes of reaction time. Journal of Experimental Psychology, 92, 103-110.

Porges, S. W. (1984). Physiological correlates of attention: A core process underlying learning disorders. Pediatric Clinics of North America, 31, 371-385.

SpInkS, J. A., BLowers, G. H., \& SHek, D. T. L. (1985). The role of the orienting response in the anticipation of information: A skin conductance response study. Psychophysiology, 22, 385-394.

SPINKS, J. A., SidDLE, D. (1983). The functional significance of the orienting response. In D. Siddle (Ed.), Orienting and habituation: Perspectives in human research (pp. 237-314). New York: Wiley.

Surwillo, W. W., \& QuILTER, R. E. (1965). The relation of frequency of spontaneous skin potential responses to vigilance and to age. Psychophysiology, 1, 272-276.

Thackray, R. I., Bailey, J. P., \& Touchstone, R. M. (1977). Physiological, subjective, and performance correlates of reported boredom and monotony while performing a simulated radar control task. In R. Mackie (Ed.), Vigilance: Theory, operational performance, and physiological correlates (pp. 203-216). New York: Plenum Press.

TURPIN, G. (1983). Unconditioned reflexes and the autonomic nervous system. In D. Siddle (Ed.), Orienting and habituation: Perspectives in human research (pp. 1-70). New York: Wiley.

WARM, J. S. (ED.). (1984). Sustained attention in human performance. New York: Wiley.

(Manuscript received July 3, 1986; revision accepted for publication November 21,1986 .) 